\title{
Lifetime Assessment of Dietary Nucleotides Consumption in Sprague-Dawley Rats
}

\author{
Meihong $\mathrm{Xu}^{1,2}$, Rui Liang ${ }^{1,2}$, Qianying Guo ${ }^{1,2}$, Shuangjia Wang ${ }^{1,2}$, Ming Zhao ${ }^{1,2}$, Zhaofeng Zhang ${ }^{1,2}$, Junbo Wang ${ }^{1,2}$, \\ Yong $\mathrm{Li}^{1,2, *}$ \\ ${ }^{1}$ Department of Nutrition and Food Hygiene, School of Public Health, Peking University, Beijing, PR China \\ ${ }^{2}$ Beijing Key Laboratory of Toxicological Research and Risk Assessment for Food Safety, Peking University, Beijing, PR China \\ *Corresponding author: liyongbmu@163.com
}

Received April 29, 2014; Revised October 24, 2014; Accepted October 26, 2014

\begin{abstract}
To assess the long-term toxicity and carcinogenicity of dietary nucleotides (NTs), Sprague-Dawley rats were administered the NTs for life-through with the dose of $0,0.01 \%, 0.04 \%, 0.16 \%$ and $0.64 \%$. With the exception of several groups, the results showed no significant differences in weight gain, clinical symptoms data, blood indicators, and Non-neoplastic lesions frequencies. NTs have dramatically increased the food consumption of both sexes $(\mathrm{P}<0.05)$. Meanwhile, NTs decreased spontaneous tumor incidence of both sexes, notably the male rats $(\mathrm{P}<0.05)$. The occurrence of all malignant and mammary tumors in NTs-treated females was lower than those in controls $(\mathrm{P}<0.05)$. In comparison with the control group, the incidence of death from malignant and systemic tumors were obviously decreased in the NTs-treated groups $(\mathrm{P}<0.01)$. Moreover, our results showed that a higher percentage of rats in the NTs-treated groups died of non-neoplastic lesions compared with control. In conclusion, dietary nucleotides are not toxic or carcinogenic in rats up to $0.64 \%$ for all the life, and $0.64 \%$ was an unobservable effect dose.
\end{abstract}

Keywords: dietary nucleotides, safety, lifelong, Spontaneous Tumor Incidence, Sprague-Dawley rat

Cite This Article: Meihong Xu, Rui Liang, Qianying Guo, Shuangjia Wang, Ming Zhao, Zhaofeng Zhang, Junbo Wang, and Yong Li, "Lifetime Assessment of Dietary Nucleotides Consumption in Sprague-Dawley Rats." Journal of Food and Nutrition Research, vol. 2, no. 11 (2014): 806-813. doi: 10.12691/jfnr-2-11-8.

\section{Introduction}

Nucleotides and their metabolic products play key roles in most of biological processes, they are base units of nucleic acids, coenzyme components and biological regulators that transferring chemical energy or in biosynthetic pathways. There is increasing evidence that dietary nucleotides affect different aspects of body function and health, including lipid metabolism [1], immune function $[2,3,4,5]$, intestinal microbiota [6], liver function and morphology [7] as well as growth performance [8]. Although nucleotides can be synthesized de novo, several tissues with a rapid turnover rate, such as the intestine, may be benefit from an exogenous supply to save energy. Additionally, nucleotides are naturally present in all foods at very low levels. Nucleotides become semi-essential nutrients, when the body at disease states, periods of limited nutrient intake or rapid growth, such as the weaning period [9].

With the multifaceted benefits of nucleotides, there is a history of safe addition of dietary nucleotides (NTs) in human diet. Previous evidence suggests that NTs are particularly beneficial for infants [10]. US Food and Drug Administration (FDA) recommends the fortification of substitutes with nucleotide in milk formula. However, the similar incidence of adverse events, between the human milk-fed and the formula-fed groups, provides presumptive evidence regarding to the safety of NTs for preterm infants. Interestingly, to our best knowledge, is no available data about the long-term, especially for the lifelong, safety of NTs. when we using rats to evaluate the safety of life-long consumption of NTs, it's convenient to assess age-related spontaneous tumor incidence and development of adverse side effects at the same time.

In spite of previous short-term studies which did not suggest carcinogenic potential of NTs [11], the potential carcinogenicity of chronic treatments is still unclear. Therefore, the effects of NTs on the lifelong safety and the spontaneous tumor development in Sprague-Dawley (S-D) rats were investigated in this study.

\section{Materials and Methods}

\subsection{Test Substance}

NTs provided by Zhen-Ao Biotechnology Ltd. Co. (Dalian, China) were derived from brew yeast RNA, whose purity is more than 99\%. The composition of NT mixture, contained adenosine monophosphate (AMP), guanosine monophosphate (GMP), uridine monophosphate (UMP) and cytidinemonophosphate (CMP), is followed the ratio, which is 5'AMP 5'CMP 5'GMPNa2 5'UMPNa2 made in accordance with 22.8: 
26.6: 30.2: 20.4. The experimental diet was supplemented with “AIN93G" to give 10, 40, 160 and $640 \mathrm{mg}$ of mixed NT kg-1 diet.

\subsection{Experimental Animal and Housing Conditions}

A total of 500 male and female S-D rats (4 weeks old), weighing 35-55 g, were obtained from the Animal Service of Health Science Center, Peking University, Beijing. Rats were housed two per plastic cages with free access to chow and tap water in a SPF (Specific Pathogen Free) filter-protected air-conditioned room with controlled temperature (25 $\pm 28 \mathrm{C})$, relative air humidity $(60 \pm 5 \%)$, and 12-hour light/dark cycles (light on 07:30-19:30 hours). All animals were handled in accordance with the guidelines of the National Institutes of Health (NIH Publication No. 85-23 revised 1985) and the guidelines of the Peking University Animal Research Committee (www.lab.pku.edu.cn).

\subsection{Experimental Design}

After a 1-week acclimation period, rats were randomly assigned to one of five groups (50 animals per sex per group): one control group and four experimental groups. Control rats were fed with rodent diet (Vital River Ltd. Co., Beijing). Rats in the four experimental groups were fed with $0.01 \%, 0.04 \%, 0.16 \%$ or $0.64 \%$ (wt/wt) NTs in the diet.

All animals were observed three times per day (at 08:00, 14:00, and 20:00 hours) for the moribundity and mortality. Observations included changes in skin, fur, eyes, mucous membranes, somatomotor activity, and behavior patterns. Body weight and food consumption were recorded weekly in the first 6 months and every 2 weeks thereafter. All animals were examined weekly for the presence of palpable tumors (including mammary and skin/ subcutaneous tumors). Special attention was paid to the palpable mass development during the study. The onset time, location, size, texture, and progression of each palpable mass were recorded throughout the study period. The onset time was defined as the date (or survival time) when the palpable mass could be palpated by three checkers and the three dimensions were larger than $5 \mathrm{~mm}$. The growth time was defined as the period from the onset of a palpable mass to death from any cause.

When the rats were 90 days, 6 months, and 12 months old, eight of them from each group were executed at random. They were anesthetized by $\mathrm{CO} 2$ inhalation and then sacrificed. Keep the others under observation until natural death or until they showed predefined "end points". The "end points" were predefined symptoms indicative of non-recoverable health problems or prolonged pain (such as rapid excessive weight loss, dehydration, inability to ambulate and obtain food and water, labored respiration, infected or necrotic tumors, and tumors that impair ability to walk with normal gait) [12]. The date of each death was recorded, and the mean overall life span and the maximal life span were evaluated.

\subsection{Biochemical Assays}

Blood samples for serum biochemical evaluation were collected from femoral artery at 12 months old, and the lateral tail vein at 24 months old. Before collecting the lateral tail vein, the animals were fasted overnight prior to the collection of blood samples. The levels of alanine aminotransferase (ALT), aspartate aminotransferase (AST), total protein (TP), albumin (ALB), serum uric acid (UA), blood urea nitrogen (BUN), creatinine (CR), total cholesterol (TC), triglyceride (TG), high-density lipoprotein-cholesterol (HDL-C) and glucose(GLU) in serum were detected by automatic biochemistry analyzer (Hitachi, Japan).

\subsection{Pathomorphological Examination}

A full necropsy was done on all animals that died spontaneously or were sacrificed in moribund status. At necropsy, all the internal organs and cavities were examined. The three-dimensional sizes of all tumors were measured with a caliper. The geometric volume of tumor was determined using the formula: length $\times$ width $\times$ height $\times 0.5326$, assuming a hemi-ellipsoidal shape [13]. All tumors, as well as the tissues and organs with suspected tumors, were excised and fixed in $10 \%$ buffered formalin. Then the tissues were embedded in paraffin, sectioned at 5 $\mathrm{mm}$ with a rotary microtome, and stained with hematoxylin and eosin for microscopic examination (Olympus BH2 microscope, Olympus Optical Co., Ltd., Tokyo). According to the recommendations of the International Agency of Research on Cancer, tumors were designated as "fatal" (those that were directly or indirectly responsible for the animal's death) or "incidental" (those that did not cause death and death had arisen from an unrelated cause) [14,15]. The primary causes of spontaneous death were determined by the symptoms in moribund status as well as the gross and histopathologic findings.

\subsection{Statistical Analysis}

Statistical analyses were performed using SPSS software (version 19.0, SPSS Inc., Chicago, IL, USA). Variances in the measurement data (i.e. serum parameters, and the size, onset time, and growth time of tumor) were checked for homogeneity by Bartlett's test. When the data were homogeneous, the one-way analysis of variance test and multiple comparison of Dunnett's t test were used. The heterogeneous cases were analyzed with the KruskalWallis rank sum test. Categorical data, like the frequency of tumor and frequency of death causes, were compared using Fisher's exact probability test. All reported P values were two-sided. A value of $\mathrm{P}<0.05$ was considered significant.

\section{Results}

\subsection{Survival and General Indicators}

The survival analysis of rats of the control and NTtreated groups was done for all animals which are and tumor-bearing animals or tumor free animals.

The mean life spans of rats treated with NTs were obviously increased compared with controls. Meanwhile, the effect of NTs on longevity was significant. When gender difference was considered, the significance was attenuated because of the smaller sample size. The overall 
mean life spans of tumor-bearing animals were $746.48 \pm 16.31$ days for males and $701.85 \pm 17.34$ days for females. Note that the tumor-free animals had a much shorter life span $(693.51 \pm 17.48$ days for males and $626.98 \pm 16.54$ days for females) compared with the tumorbearing animals. The mean life spans of tumor-bearing rats treated with NTs were dose-dependently prolonged compared with the controls, with significance in the $0.01 \%, 0.04 \%$ and $0.64 \%$ NT-treated group of both sexes. As for the tumor-free rats, the significant longevity extension effect was not observed neither in the NTtreated group nor female groups.

There was no remarkable difference in general condition and behavior between the control and NTtreated rats of both sexes.

\subsection{Effects on Safety Indicators}

There were no significant differences in body weight among groups of either sex throughout the experimental period. Only at the age of 1 and 3 months was the body weight of NT-treated groups significantly heavier than the control group $(\mathrm{P}<0.05)$, while no does-related change was indicated. However, there was NTs remarkable change in food consumption in either sex throughout the experiment. During the entire period of observation, significant difference in food consumption was indicated from the comparison, in which the food consumption of NT-treated were higher than the control group $(\mathrm{P}<0.05)$, while no does-related change was indicated. At the age of 12 and 24 months, the food consumption of NT-treated female groups have no significant differences between the control group and the NT-treated groups. Organ/bodyweight ratios were unaffected by NTs (data not shown, for simplicity).

Comparison of mean serum chemistry values from the NTs treated groups with the value from normal control was show on Supplementary_material 1 and 2. Analyzing the 12-month data, the changed were shown as follows. The significant differences of ALT, compared with control, were observed in the $0.01 \%$ NTs-treated male group; meanwhile, the significant differences of AST was observed in the $0.04 \%$ and $0.64 \%$ NTs-treated male group $(\mathrm{P}<0.05)$. The significant differences of TP and ALB were observed in the $0.16 \%$ and $0.64 \%$ NTs-treated both male and female groups $(\mathrm{P}<0.05)$; meanwhile, the significant differences of $\mathrm{Cr}$, compared with control, were only observed in the $0.04 \%$ NTs-treated male group $(\mathrm{P}<0.05)$. The serum of lipid was remarkable changed in TC of $0.04 \%$ NTs-treated and TG of $0.16 \%$ and $0.64 \%$ NTstreated male groups $(\mathrm{P}<0.05)$. The serum of glucose was remarkable decreased in $0.01 \%$ and $0.16 \%$ NTs-treated male groups, but increased in $0.01 \%$ and $0.04 \%$ NTstreated female groups $(\mathrm{P}<0.05)$. As for $24-$ month data, there was only one remarkable differences between $0.64 \%$ NTs-treated female group with control in UA $(\mathrm{P}<0.05)$.

\subsection{Neoplastic Lesions}

The presence of neoplastic lesions at anatomic sites was revealed by macroscopic and histological analyses (Table 1 and Table 2). Treatment with NTs exerted a certain inhibitory effect on spontaneous carcinogenesis in rats, which was manifested by a decreased incidence of total tumors in both sexes (Supplementary_material 3 and 4). Compared with the control group, significantly reduced overall tumor incidences were observed in the NTs-treated male groups (Fisher's exact test, $\chi 2=17.02, \mathrm{P}<0.01$ ).

Table 1. Spontaneous neoplastic lesions in Sprague-Dawley female rats treated with different levels of dietary nucleotides

\begin{tabular}{|c|c|c|c|c|c|c|}
\hline \multirow{2}{*}{ Female } & \multirow{2}{*}{ Lesion } & \multicolumn{5}{|c|}{$\operatorname{NTs}(\%)$} \\
\hline & & $0(n=26)$ & $0.01(n=26)$ & $0.04(n=26)$ & $0.16(n=26)$ & $0.64(n=26)$ \\
\hline \multirow{8}{*}{ Mammary gland } & Adenoma (B) & $6(10)$ & 5 & 4 & $6(7)$ & $7(9)$ \\
\hline & Fibroadenoma (B) & $3(4)$ & 2 & 2 & $4(5)$ & 3 \\
\hline & Benign tumor size/ rat $(\mathrm{cm} 3)$ a & $194.8 \pm 138.60$ & $143.63 \pm 28.82$ & $143.23 \pm 32.33$ & $180.69 \pm 28.53$ & $208.21 \pm 21.74$ \\
\hline & Adenocarcinoma (M) & 2 & 0 & 0 & 0 & 1 \\
\hline & Malignant tumor size/rat $(\mathrm{cm} 3)$ a & $230.25 \pm 52.86$ & - & - & - & 203.11 \\
\hline & Total tumor size/rat $(\mathrm{cm} 3)$ a & $202.77 \pm 41.12$ & $143.63 \pm 28.82 *$ & $143.23 \pm 32.33$ & $180.69 \pm 28.53$ & $207.81 \pm 20.86$ \\
\hline & Onset time (day) & $532.8 \pm 64.1$ & $615.9 \pm 114.1^{*}$ & $580.2 \pm 38.2 *$ & $587.5 \pm 127.2$ & $572.3 \pm 75.2$ \\
\hline & Growth time (day) a & $212.4 \pm 81.1$ & $241.0 \pm 56.2^{*}$ & $183.8 \pm 73.9$ & $193.9 \pm 45.8$ & $212.7 \pm 50.0$ \\
\hline \multirow[t]{6}{*}{ Skin/Subcutis } & Adenoma (B) & 1 & 0 & 0 & 0 & 0 \\
\hline & Fibroma (B) & 2 & 1 & 1 & 0 & 1 \\
\hline & Lipoma (B) & 0 & 0 & 0 & 0 & 1 \\
\hline & Total tumor size/rat $(\mathrm{cm} 3)$ a & $78.51 \pm 38.91$ & 67.19 & 81.23 & - & $74.47 \pm 86.56$ \\
\hline & Onset time (day) & $579.1 \pm 51.6$ & 657 & 662 & - & $634.9 \pm 88.7$ \\
\hline & Growth time (day) a & $131.8 \pm 80.5$ & 113 & 163 & - & $122.7 \pm 90.4$ \\
\hline \multirow[t]{2}{*}{ Pituitary gland } & Adenoma (B) & $17(14)$ & $8(7)$ & $10(8)$ & $11(9)$ & $12(10)$ \\
\hline & Total tumor size/rat $(\mathrm{cm} 3)$ a & $0.39 \pm 0.11$ & $0.35 \pm 0.13$ & $0.37 \pm 0.13$ & $0.34 \pm 0.09$ & $0.38 \pm 0.11$ \\
\hline \multirow[t]{2}{*}{ Liver } & Adenoma (B) & 0 & 2 & 0 & 0 & 0 \\
\hline & Tumor size/rat (cm3) a & - & $3.62 \pm 0.50$ & - & - & - \\
\hline \multirow[t]{2}{*}{ Lung } & Lung metastases (M) & 1 & 0 & 0 & 0 & 1 \\
\hline & Tumor size/rat (cm3) a & - & - & - & - & 0.21 \\
\hline \multirow[t]{2}{*}{ Pancreas } & Adenocarcinoma (M) & 1 & 0 & 0 & 1 & 1 \\
\hline & Tumor size/rat (cm3) a & 9.1 & - & - & 8.63 & 8.47 \\
\hline \multirow[t]{4}{*}{ Ovarian } & Luteinoma (B) & 0 & 1 & 0 & 1 & 0 \\
\hline & Tumor size/rat (cm3) a & - & 0.41 & - & 0.36 & - \\
\hline & Malignant teratoma (M) & 0 & 0 & 0 & 0 & 1 \\
\hline & Tumor size/rat (cm3) a & - & - & - & - & 0.67 \\
\hline \multirow[t]{2}{*}{ Abdominal cavity } & Vascular tumor (M) & 1 & 1 & 0 & 1 & 0 \\
\hline & Tumor size/rat $(\mathrm{cm} 3)$ a & 179.2 & 187.3 & - & 191.7 & - \\
\hline
\end{tabular}

Benigntumor (B) andmalignant tumor (M).

Data are the number of rats with the lesions where the number is parentheses is the total number of lesions in the group when at least one rat has more than one lesion, or ${ }^{\mathrm{a}}$ mean \pm SD.

Significant difference compared with the control group (by one-way analysis of variance): ${ }^{*} \mathrm{P}<0.05$ 
Table 2. Spontaneous neoplastic lesions in Sprague-Dawley male rats treated with different levels of dietary nucleotides

\begin{tabular}{|c|c|c|c|c|c|c|}
\hline \multirow{2}{*}{ Male } & \multirow{2}{*}{ Lesion } & \multicolumn{5}{|c|}{ NTs(\%) } \\
\hline & & $0(n=26)$ & $0.01(n=26)$ & $0.04(n=26)$ & $0.16(n=26)$ & $0.64(n=26)$ \\
\hline \multirow[t]{5}{*}{ Skin/Subcutis } & Adenoma (B) & 2 & 3 & 3 & 0 & 3 \\
\hline & Fibroma (B) & 4 & 2 & 3 & 2 & 2 \\
\hline & Total tumor size/rat $\left(\mathrm{cm}^{3}\right)^{\mathrm{a}}$ & $77.82 \pm 33.62$ & $72.25 \pm 33.93$ & $89.86 \pm 61.04$ & $86.27 \pm 48.23$ & $72.98 \pm 25.04$ \\
\hline & Onset time (day) ${ }^{\mathrm{a}}$ & $530.5 \pm 52.4$ & $614.8 \pm 63.6^{*}$ & $578.8 \pm 40.3$ & $634.8 \pm 28.2$ & $642.7 \pm 43.9$ \\
\hline & Growth time (day) ${ }^{a}$ & $239.9 \pm 40.5$ & $205.5 \pm 39.0$ & $271.5 \pm 66.0$ & $234.0 \pm 68.8$ & $228.9 \pm 56.9$ \\
\hline \multirow[t]{2}{*}{ Pituitary gland } & Adenoma (B) & $9(7)$ & $4(3)$ & $4(3)$ & 4 & $4(3)$ \\
\hline & Tumor size/rat $\left(\mathrm{cm}^{3}\right)^{\mathrm{a}}$ & $0.40 \pm 0.14$ & $0.31 \pm 0.05$ & $0.30 \pm 0.17$ & $0.39 \pm 0.10$ & $0.43 \pm 0.10$ \\
\hline \multirow[t]{2}{*}{ Liver } & Adenoma (B) & 1 & 1 & 1 & 0 & 1 \\
\hline & Tumor size/rat $\left(\mathrm{cm}^{3}\right)^{\mathrm{a}}$ & 1.21 & 1.05 & 1.39 & - & 0.87 \\
\hline \multirow[t]{2}{*}{ Lung } & Lung metastases (M) & 1 & 0 & 0 & 0 & 0 \\
\hline & Tumor size/rat $\left(\mathrm{cm}^{3}\right)^{\mathrm{a}}$ & 0.12 & - & - & - & - \\
\hline \multirow[t]{2}{*}{ Pancreas } & Adenocarcinoma (M) & 0 & 0 & 0 & 1 & 2 \\
\hline & Tumor size/rat $\left(\mathrm{cm}^{3}\right)$ & & & & 10.62 & $13.04 \pm 1.12$ \\
\hline \multirow[t]{3}{*}{ Adrenal gland } & Cortical adenoma (B) & 0 & 1 & 0 & 0 & 0 \\
\hline & Tumor size/rat $\left(\mathrm{cm}^{3}\right)$ & & 0.18 & - & - & - \\
\hline & Tumor size/rat $\left(\mathrm{cm}^{3}\right)^{\mathrm{a}}$ & - & - & - & 0.08 & - \\
\hline \multirow[t]{2}{*}{ Prostate } & Adenoma(B) & 1 & 1 & 1 & 2 & 1 \\
\hline & Tumor size/rat $\left(\mathrm{cm}^{3}\right)^{\mathrm{a}}$ & 2.67 & 2.38 & 1.76 & $2.81 \pm 0.91$ & 2.00 \\
\hline \multirow[t]{2}{*}{ Bladder } & Cell carcinoma (M) & 1 & 0 & 1 & 0 & 0 \\
\hline & Tumor size/rat $\left(\mathrm{cm}^{3}\right)^{a}$ & 0.02 & - & 0.04 & - & - \\
\hline \multirow[t]{2}{*}{ Abdominal cavity } & Vascular tumor (M) & 1 & 0 & 0 & 0 & 1 \\
\hline & Tumor size/rat $\left(\mathrm{cm}^{3}\right)^{\mathrm{a}}$ & 156.7 & - & - & - & 134.1 \\
\hline Systemic & Lymphoma (M) & 2 & 0 & 0 & 0 & 0 \\
\hline
\end{tabular}

Benign tumor (B) and malignant tumor (M).

Data are the number of rats with the lesions where the number is parentheses is the total number of lesions in the group when at least one rat has more than one lesion, or ${ }^{\mathrm{a}}$ mean $\pm \mathrm{SD}$

Significant difference compared with the control group (by Fisher's exact probability test):* $\mathrm{P}<0.05$

The results showed that NTs did not increase the frequency of malignant or benign tumors. Moreover, the occurrence of benign and malignant tumors in all NTstreated groups of both sexes tended to decrease compared with the control (Supplementary_material 3 and 4). Disregarding the division by sex, males treated by $0.01 \%$, $0.04 \%, 0.16 \%$ and $0.64 \%$ NTs resulted in $10-, 10-, 2.5$-fold and twice decreases in the malignant tumor incidence compared with the control (Fisher's exact tests, $\chi 2=13.247$, $\mathrm{P}=0.01$,).

Mammary tumors, the most frequent neoplastic lesions in female S-D rats, were present in eight (31\%) control females. Animals with mammary tumors accounted for $30 \%, 23 \%, 35 \%$ and $31 \%$ of the $0.01 \%, 0.04 \%, 0.16 \%$ and $0.64 \%$ NTs-treated rats, respectively, which indicated no changed tendency of mammary tumor incidence. Remarkable difference was found in the onset time of mammary tumor between the $0.01 \%$ NTs- and controltreated female rats $(\mathrm{P}<0.05)$. At the time of necropsy, the volume of the mammary tumor per rat in the $0.01 \%$ and $0.04 \%$ NTs-treated groups was significantly decreased compared with the control females $(\mathrm{P}<0.05)$. In addition, the average growth time of the mammary tumor per rat in the $0.01 \%$ NTs-treated groups tended to be longer than that of control rats. Consistent with the mammary tumor results, the skin/subcutaneous tumors in NTs-treated rats at necropsy exhibited a lateness onset time compared to the controls $(\mathrm{P}<0.05)$.

Pituitary tumor is a frequent neoplastic lesion in S-D rats of both sexes. Our results showed that the incidences of pituitary gland adenoma in the control group were $31.3 \%$ in males and $63.6 \%$ in females. Rats treated with NTs exhibited decreases in the incidence of pituitary gland adenomas compared with the control rats in either sex. At the time of necropsy, there was no marked difference in the volume of pituitary tumor between NTs-treated groups and the control groups of either sex.

\subsection{Non-neoplastic Lesions}

Hepatic steatosis, the most frequent non-neoplastic lesion observed, occurred in 5 control (19.2\%) and 3 (11.5\%), 4 (15.4\%), 4 (15.4\%) and 3 (11.5\%) males treated with $0.01 \%, 0.04 \%, 0.16 \%$ and $0.64 \%$ NTs, respectively, and in 4 control (15.4\%) and $3(11.5 \%), 4$ $(15.4 \%) 3(11.5 \%)$ and $4(15.4 \%)$ females treated with $0.01 \%, 0.04 \%, 0.16 \%$ and $0.64 \%$ NTs. Also, several inflammatory lesions were detected, all at low frequencies without differences between the treated and control.

Among the rats bearing nonfatal tumor or the tumorfree rats, the deaths were mainly attributed to vital organ failure caused by age-related non-neoplastic lesions, such as chronic nephropathy, hepatocirrhosis, and emphysema. Our results showed that a higher percentage of rats in the NTs-treated groups died of non-neoplastic lesions compared with control.

\subsection{Probable Causes of Spontaneous Deaths}

The causes of spontaneous death were summarized into two categories: neoplastic and non-neoplastic lesions. It should be noted that some cases of spontaneous death with causes involved with a complex interaction of multiple pathologies were defined as undetermined causes. The percentage of undetermined death cause did not differ significantly among groups $(\mathrm{P}>0.05)$. The cause of death analysis had been established in more than $80 \%$ of the cases. (Supplementary_material 5 and 6)

In all groups, the main causes of death were neoplastic lesions, accounting for $69 \%, 31 \%, 19 \%, 35 \%$ and $46 \%$ of deaths in the control and NTs-treated groups, respectively, among males and $62 \%, 38 \%, 31 \%, 54 \%$ and $62 \%$ respectively, among females. Significant difference compared with the control group (by Fisher's exact probability test) was positive in $0.01 \%, 0.04 \%$ and $0.16 \%$ 
NTs $(\mathrm{P}<0.0125)$. These tumors were benign in approximately $68 \%$ of the cases, which mainly consisted of pituitary and mammary/subcutaneous adenomas. Pituitary tumor was found to be the most common neoplastic cause of death in S-D rats for males (23\%) and females (19\%) in the control group. As described in previous studies, pituitary tumors are fatal in S-D rats when the mean diameter of the lesions exceeds $7 \mathrm{~mm}$ because the large intracranial mass produces cerebral compression. Compared with the control group, the frequency of death due to pituitary tumor in the NTstreated groups exhibited a decreased tendency when genders were pooled.

For female rats, mammary gland tumor was the second most common cause of death. Additionally, a few cases of skin/subcutaneous tumors were lethal for both sexes. The mammary gland tumor as well as skin/subcutaneous tumor might result in a moribund condition of the individual when the large mass had impeded feeding and/or ulcerated and then underwent necrosis. Compared with the control group, the frequencies of the large masses of mammary or skin/subcutaneous benign tumors as causes of death were decreased to some extent in NTs-treated rats.

As another type of cause of neoplastic death, malignant and systemic (leukemia and lymphoma) tumors were fatal by invasion of or metastasis to vital organs. Compared with the control groups, the incidences of death from malignant and systemic tumors in the NTs-treated groups of both sexes were obviously decreased (Fisher's exact test, $\chi 2=14.764, \mathrm{P}=0.005$ ).

\section{Discussion}

With their multifaceted activities, NTs have gained increasing popularity as dietary supplements. This study aims to provide evidence to assess the long term safety of NTs supplementation. This lifelong S-D rats study indicated that at up to $0.64 \%$ dose, NTs have no adverse effects. There was also no evidence of target organ toxicity. In contrast, the results of our study revealed that NTs inhibit the incidence and development of spontaneous tumors in S-D rats.

We analyzed the whole blood indicators (TP, ALB, CR and so on) and most of them were similar between groups, indicating no clinical adverse effect changes of liver and kidney function in NTs-treated groups throughout the experiment. It must be noticed that the blood of rats was collected at the time points of their 12 and 24 months old. Because of that, some Ns are 4 and some are 8 .

Our previous study showed long-term administration of NTs increased the survival and prolonged the life span of rats, especially for the long-lived sub-population [16]. Since cancer is one of the top reasons of death, this time we evaluated the spontaneous carcinogenesis.

Inhibitory effects of NTs on the spontaneous carcinogenesis were revealed in our study. The age-related spontaneous tumor incidences in control rats, $80.1 \%$ in females and $69.2 \%$ in males, were close to the reported frequencies of $87.0-95.8 \%$ and $70.0-76.7 \%$ for aged female and male S-D rats, respectively [17]. In the present study, pituitary gland adenomas and mammary adenomas developed most frequently in the control rats, which is consistent to the oncological characteristics of S-D rats
$[18,19]$. Decreasing tendencies were observed in the incidences of pituitary gland and mammary gland tumors in NTs-treated rats. However, the significance of these findings still need to be confirmed by a larger sample size. Most interestingly, our result showed both postponed onset time and increased growth time of the palpable tumors, including mammary tumors and skin/subcutaneous tumors, implied that administration of NTs might inhibit the development and the growth rate of existing tumors. In contrary to the result, previous study demonstrated supplementation of a nucleotide-free diet with a nucleoside-nucleotides mixture prevents of nonneoplastic lesions, such as amyloidosis, without promoting tumor growth in 252Cf-irradiated mice [3]. In accordance with those result, we found a decreased death rate caused by mammary or skin/subcutaneous benign tumors in NT-treated groups, which further supports the inhibitory effect of NTs on the tumor development and lethality. All in all, the influence of NTs on total tumor incidence, malignancy rate as well as tumor development might be the major reason of the prolonged life in NTstreated groups.

Aging-related diseases (including cancer) are positively related with high body weight induced by excessive energy consumption [20]. However, the NTs didn't have any impact on the body weight of the animals.

In our previous studies, NTs were observed to exert a considerably beneficial effect on the age-related oxidative status, namely, the inhibition of the age-related decrease of the activity of antioxidant enzymes, superoxide dismutase (SOD) and glutathione peroxidase (GSH-PX), and increase of Malondialdehyde (MDA). Meanwhile, another study by our group suggested administration of $0.01 \%, 0.04 \%, 0.16 \%$ and $0.64 \%$ NTs for 3 months could dose-dependently increase the activity of superoxide dismutase (SOD) and glutathione peroxidase (GSH-PX) and decrease the level of MDA in aged C57BL/6J mice.

As we know, MDA is a potentially important contributor to DNA damage and mutation and is verified to be carcinogenic in rats [21]. Disturbances of oxidative status will weaken defense systems and increased peroxides can lead to age-related degenerative diseases as well as cancer [22]. Lifelong reduction in SOD activity leads to DNA damage and in turn, cancer [23]. Moreover, it is confirmed that antioxidants or the free radical scavengers are be the inhibitors at both initiation and during the promotion/transformation stage of carcinogenesis by protecting cells against oxidative damage [24,25]. For this reason, we suggest that the protection by NTs against age-related oxidative stress may contribute to the effect on inhibiting the incidence of spontaneous tumors and prolonging the life span in S-D rats.

It must be noted the drawback of experimental design. The experiment began in 2008, when the OECD had not published Test Guideline 453 (Combined Chronic ToxicitylCarcinogenicity studies). And it has taken years for the observation of rats. According to above reason, the present study was lack of adherence to safety guidelines OECD 453. The point should be done in further research.

\section{Conclusions}


In conclusion, a chronic/carcinogenicity study of NTs reveals that lifelong supplement at dose as high as $0.64 \%$ through lifelong time did not show any evidence of target organ toxicity. The NOAEL is considered to be $0.64 \%$. Moreover, our data indicates that NTs administered throughout the life span have significant influence on spontaneous tumor onset time and development, death rate in $\mathrm{S}-\mathrm{D}$ rats by their anti-oxidative activity.

\section{Acknowledgement}

The authors are grateful to Zhen-Ao Biotechnology Ltd. for providing the samples of dietary nucleotides used in this study.

\section{References}

[1] Fontana L, Moreira E, Torres MI, et al. Dietary nucleotides correct plasma and liver microsomal fatty acid alterations in rats with liver cirrhosis induced by oral intake of thioacetamide. J Hepatol. 1998; 28 (4): 662-9.

[2] Jyonouchi H, Sun S, Winship T, et al. Dietary ribonucleotides increase antigen-specific type 1 T-helper cells in the regional draining lymph nodes in young BALB/cJ mice. Nutrition. 2003; 19 (1): 41-6.

[3] Yokoyama H, Fujiwara H, Watanabe H. Dietary nucleosides and nucleotides do not affect tumor incidence but reduce amyloidosis incidence in B6C3F1 mice irradiated with californium- 252 . Nutrition. 2004; 20 (4): 383-9.

[4] Frankic T, Pajk T, Rezar V, et al. The role of dietary nucleotides in reduction of DNA damage induced by $\mathrm{T}-2$ toxin and deoxynivalenol in chicken leukocytes. Food Chem Toxicol. 2006; 44 (11): 1838-44.

[5] Tahmasebi KA, Keyvanshokooh S, Nematollahi A, et al. Dietary administration of nucleotides to enhance growth, humoral immune responses, and disease resistance of the rainbow trout (Oncorhynchus mykiss) fingerlings. Fish Shellfish Immunol. 2011; 30 (1): 189-93.

[6] Sauer N, Bauer E, Vahjen W,et al. Nucleotides modify growth of selected intestinal bacteria in vitro. Livestock Science. 2010; 133 (1): $161-3$.

[7] Lopez-Navarro AT, Ortega MA, Peragon J, et al. Deprivation of dietary nucleotides decreases protein synthesis in the liver and small intestine in rats. Gastroenterology. 1996; 110 (6): 1760-9.

[8] Iwasa $\mathrm{Y}$, Iwasa $\mathrm{M}$, Ohmori $\mathrm{Y}$, et al. The effect of the administration of nucleosides and nucleotides for parenteral use. Nutrition. 2000; 16 (7-8): 598-602.

[9] Sanchez PA, Gil A. Nucleotides as semiessential nutritional components. Br J Nutr 2002; 87 (Suppl 1): S135-7.

[10] Singhal A, Kennedy K, Lanigan J, et al. Dietary Nucleotides and Early Growth inFormula-Fed Infants: A Randomized Controlled Trial. Pediatrics. 2010; 126 (4): e946-53.

[11] Anisimov VN. Life span extension and cancer risk: myths and reality. Exp Gerontol. 2001; 36 (7): 1101-36.

[12] Cavigelli SA, Bennett JM, Michael KC, et al. Female temperament, tumor development and life span: relation to glucocorticoid and tumor necrosis factor alpha levels in rats. Brain Behav Immun. 2008; 22 (5):727-35.

[13] Mesia-Vela S, Sanchez RI, Roberts KG, et al. Dietary clofibrate stimulates the formation and size of estradiol-induced breast tumors in female August-Copenhagen Irish (ACI) rats. Toxicology. 2008; 246 (1): 63-7.

[14] Gart J, Krewski D, Lee PN, et al. IARC Scientific Publications Number 79: Statistical Methods in Cancer Research, Vol. III-The Design and Analysis of Long-Term Animal Experiments. International Agency for Research on Cancer, Lyon, France.

[15] Peto R, Pike MC, Day NE, et al. Guidelines for simple, sensitive significance tests for carcinogenic effects in long-term animal experiments. IARC Monogr Eval Carcinog Risk Chem Hum Suppl. 1980; (2 Suppl): 311-426.

[16] Meihong Xu, Rui Liang, Qianying Guo, et al. Dietary nucleotides Extend the Life Span in Sprague-Dawley Rat. The Journal of Nutrition, Health and Aging. 2013; 17 (3): 223-9.

[17] Baldrick P. Carcinogenicity evaluation: comparison of tumor data from dual control groups in the Sprague-Dawley rat. Toxicol Pathol. 2005; 33 (2): 283-91.

[18] Nakazawa M, Tawaratani T, Uchimoto H, et al. Spontaneous neoplastic lesions in aged Sprague-Dawley rats. Exp Anim. 2001; 50 (2): 99-103.

[19] Baldrick P, Reeve L. Carcinogenicity evaluation: comparison of tumor data from dual control groups in the CD-1 mouse. Toxicol Pathol. 2007; 35 (4): 562-9.

[20] Dirx MJ, Zeegers MP, Dagnelie PC, et al. Energy restriction and the risk of spontaneous mammary tumors in mice: a meta-analysis. Int J Cancer. 2003; 106 (5): 766-70.

[21] Inal ME, Kanbak G, Sunal E. Antioxidant enzyme activities and malondialdehyde levels related to aging. Clin Chim Acta. 2001; 305 (1-2): 75-80.

[22] Niedernhofer LJ, Daniels JS, Rouzer CA, et al. Malondialdehyde, a product of lipid peroxidation, is mutagenic in human cells. J Biol Chem. 2003; 278 (33): 31426-33.

[23] Ames BN, Shigenaga MK, Hagen TM. Oxidants, antioxidants, and the degenerative diseases of aging. Proc Natl Acad Sci USA. 1993; 90 (1): 7915-22.

[24] Sun Y. Free radicals, antioxidant enzymes, and carcinogenesis. Free Radic Biol Med. 1990; 8 (6): 583-99.

[25] Van RH, Ikeno Y, Hamilton M, et al. Life-long reduction in MnSOD activity results in increased DNA damage and higher incidence of cancer but does not accelerate aging. Physiol Genomics. 2003; 16 (1): 29-37.

\section{Supp}

Table 1. The effect of long-term administration of NTs on the serum biochemisty parameters of SD rats: 12-monthdata (mean \pm SD)

\begin{tabular}{|c|c|c|c|c|c|c|c|c|c|c|c|c|c|}
\hline Sex & $\begin{array}{l}\text { NTs } \\
(\%)\end{array}$ & $\mathrm{N}$ & $\begin{array}{l}\text { ALT } \\
(\mathrm{U} / \mathrm{L}) \\
\end{array}$ & $\begin{array}{l}\text { AST } \\
(\mathrm{U} / \mathrm{L}) \\
\end{array}$ & $\begin{array}{c}\mathrm{TP} \\
(\mathrm{g} / \mathrm{L}) \\
\end{array}$ & $\begin{array}{l}\text { ALB } \\
(\mathrm{g} / \mathrm{L})\end{array}$ & UA & $\begin{array}{c}\mathrm{BUN} \\
(\mathrm{mmol} / \mathrm{L})\end{array}$ & $\begin{array}{c}\mathrm{Cr} \\
(\mu \mathrm{mol} / \mathrm{L}) \\
\end{array}$ & $\begin{array}{c}\mathrm{TC} \\
(\mathrm{mmol} / \mathrm{L}) \\
\end{array}$ & $\begin{array}{c}\text { TG } \\
(\mathrm{mmol} / \mathrm{L})\end{array}$ & $\begin{array}{c}\mathrm{HDL} \\
(\mathrm{mmol} / \mathrm{L})\end{array}$ & $\begin{array}{c}\text { Glu } \\
(\mathrm{mmol} / \mathrm{L})\end{array}$ \\
\hline \multirow[t]{5}{*}{ Male } & 0 & 8 & $60.6 \pm 10.4$ & $194.0 \pm 32.1$ & $83.2 \pm 4.8$ & $42.1 \pm 1.0$ & $90.43 \pm 23.94$ & $6.00 \pm 0.48$ & $43.4 \pm 3.7$ & $2.05 \pm 0.85$ & $2.60 \pm 0.59$ & $1.97 \pm 0.50$ & $7.98 \pm 0.87$ \\
\hline & 0.01 & 8 & $75.9 \pm 17.8^{*}$ & $209.4 \pm 29.3$ & $82.9 \pm 2.5$ & $42.0 \pm 0.9$ & $88.23 \pm 29.10$ & $6.31 \pm 0.53$ & $43.0 \pm 5.3$ & $2.07 \pm 0.65$ & $2.45 \pm 0.34$ & $1.74 \pm 0.26$ & $6.47 \pm 0.80 *$ \\
\hline & 0.04 & 8 & $57.0 \pm 16.6$ & $142.6 \pm 17.6^{*}$ & $82.6 \pm 3.8$ & $41.1 \pm 1.3$ & $108.15 \pm 37.23$ & $5.77 \pm 0.46$ & $38.1 \pm 3.7 *$ & $3.78 \pm 1.34 *$ & $2.63 \pm 0.73$ & $1.89 \pm 0.38$ & $8.00 \pm 0.73$ \\
\hline & 0.16 & 8 & $4 \pm$ & 26.8 & $.9 *$ & $48.2 \pm 2.8^{*}$ & 19 & $18 \pm 1.20$ & $1 \pm 4.5$ & $8 \pm 1.60$ & $40 *$ & 0.30 & $6.53 \pm 0.94 *$ \\
\hline & 0.64 & 8 & $53.9 \pm 10.4$ & $163.8 \pm 31.1^{*}$ & $87.9 \pm 3.1 *$ & $47.3 \pm 2.1 *$ & $104.01 \pm 34.27$ & $5.72 \pm 1.00$ & $40.1 \pm 4.3$ & $1.11 \pm 0.60$ & $1.95 \pm 0.46^{*}$ & $1.73 \pm 0.42$ & $7.35 \pm 0.88$ \\
\hline \multirow[t]{5}{*}{ Female } & 0 & 8 & $60.6 \pm 17.0$ & $196.4 \pm 17.5$ & $81.2 \pm 3.0$ & $41.3 \pm 1.1$ & $86.14 \pm 23.06$ & $5.80 \pm 0.61$ & $43.0 \pm 6.7$ & $2.57 \pm 1.08$ & $2.35 \pm 0.30$ & $1.72 \pm 0.29$ & $6.78 \pm 0.87$ \\
\hline & 0.01 & 8 & $62.1 \pm 14.1$ & $165.6 \pm$ & $81.4 \pm 3.6$ & 6 & 80.7 & $5.76=$ & $35.4 \pm 3.6$ & $3.03 \pm$ & $2.50 \pm 0.63$ & 0.39 & $8.23 \pm 1.35 *$ \\
\hline & 0.04 & 8 & 71 & 4 & 1 & 2 & 8 & 74 & 3 & 2.76 & .67 & $1.63 \pm 0.53$ & $8.32 \pm 0.48^{*}$ \\
\hline & 0.16 & 8 & $70.3 \pm 21.4$ & $184.0 \pm 38.4$ & $89.9 \pm 7.0 *$ & $46.8 \pm 3.6^{*}$ & $78.70 \pm 23.09$ & $6.68 \pm 1.18$ & $44.4 \pm 2.5$ & $1.87 \pm 0.74$ & $2.49 \pm 0.44$ & $2.09 \pm 0.43$ & $6.85 \pm 0.71$ \\
\hline & 0.64 & 8 & $8 \pm 9.6$ & $186.6 \pm 33.6$ & $90.5 \pm 3.8^{*}$ & $47.3 \pm 1.3^{*}$ & $80.63 \pm 26.03$ & $6.19 \pm 0.82$ & $42.9 \pm 5.1$ & $1.50 \pm 0.91$ & $2.39 \pm 0.58$ & $1.99 \pm 0.50$ & $7.11 \pm 0.66$ \\
\hline
\end{tabular}

Alanine aminotransferase (ALT), aspartate aminotransferase (AST), total protein (TP), albumin (ALB), serum uric acid (UA), blood urea nitrogen

(BUN), creatinine (CR), total cholesterol (TC), triglyceride (TG), high-density lipoprotein-cholesterol (HDL-C) and glucose(GLU)

*. Statistical significance: $\mathrm{p}<0.05$, compared with normal control. 
Table 2. The effect of long-term administration of NTs on the serum biochemisty parameters of SD rats: 24-month data (mean \pm SD)

\begin{tabular}{|c|c|c|c|c|c|c|c|c|c|c|c|c|c|c|}
\hline Sex & Sex & $\begin{array}{l}\text { NTs } \\
\text { (\%) }\end{array}$ & $\mathrm{N}$ & $\begin{array}{l}\text { ALT } \\
\text { (U/L) }\end{array}$ & $\begin{array}{l}\text { AST } \\
\text { (U/L) }\end{array}$ & $\begin{array}{c}\mathrm{TP} \\
(\mathrm{g} / \mathrm{L})\end{array}$ & $\begin{array}{l}\text { ALB } \\
\text { (g/L) }\end{array}$ & UA & $\begin{array}{c}\text { BUN } \\
(\mathrm{mmol} / \mathrm{L})\end{array}$ & $\begin{array}{c}\mathrm{Cr} \\
(\mu \mathrm{mol} / \mathrm{L})\end{array}$ & $\begin{array}{c}\mathrm{TC} \\
(\mathrm{mmol} / \mathrm{L})\end{array}$ & $\begin{array}{c}\mathrm{TG} \\
(\mathrm{mmol} / \mathrm{L})\end{array}$ & $\begin{array}{c}\mathrm{HDL} \\
(\mathrm{mmol} / \mathrm{L})\end{array}$ & $\begin{array}{c}\text { Glu } \\
(\mathrm{mmol} / \mathrm{L})\end{array}$ \\
\hline \multirow[t]{4}{*}{ Male } & $\sigma^{\prime \prime}$ & 0 & 5 & $23.77 \pm 18.01$ & $89.00 \pm 41.58$ & $51.33 \pm 21.08$ & $27.33 \pm 11.50$ & $67.67 \pm 25.48$ & $16.67 \pm 14.01$ & $88.67 \pm 56.92$ & $1.93 \pm 0.06$ & $0.73 \pm 0.58$ & $0.87 \pm 0.06$ & $6.47 \pm 1.78$ \\
\hline & $\sigma^{\prime \prime}$ & 0.01 & 4 & $37.33 \pm 16.86$ & $116.33 \pm 5.53$ & $47.00 \pm 10.82$ & $26.00 \pm 6.25$ & $60.33 \pm 16.29$ & $4.97 \pm 1.12$ & $32.00 \pm 45.83$ & $2.73 \pm 0.83$ & $0.60 \pm 0.10$ & $1.57 \pm 0.59$ & $6.50 \pm 1.35$ \\
\hline & $\sigma^{\prime \prime}$ & 0.04 & 5 & $32.80 \pm 15.70$ & $110.00 \pm 42.97$ & $49.60 \pm 8.65$ & $28.20 \pm 4.38$ & $79.60 \pm 23.00$ & $6.36 \pm 1.85$ & $53.00 \pm 33.26$ & $1.86 \pm 0.55$ & $0.80 \pm 0.45$ & $1.10 \pm 0.42$ & $6.54 \pm 3.18$ \\
\hline & $\sigma^{\prime \prime}$ & 0.16 & 4 & $36.25 \pm 26.041$ & $102.25 \pm 61.13$ & $46.00 \pm 11.17$ & $25.50 \pm 5.92$ & $57.50 \pm 16.84$ & $4.30 \pm 1.01$ & $33.50 \pm 65.57$ & $1.50 \pm 0.36$ & $0.25 \pm 0.50$ & $0.90 \pm 0.16$ & $5.68 \pm 3.21$ \\
\hline & $\sigma^{n}$ & 0.64 & 7 & $30.00 \pm 19.24$ & $108.14 \pm 4.72$ & $54.29 \pm 7.72$ & $27.29 \pm 8.99$ & $70.14 \pm 17.35$ & $15.03 \pm 11.18$ & $82.00 \pm 58.39$ & $1.53 \pm 0.93$ & $1.00 \pm 0.82$ & $0.91 \pm 0.43$ & $5.23 \pm 2.96$ \\
\hline
\end{tabular}

Female

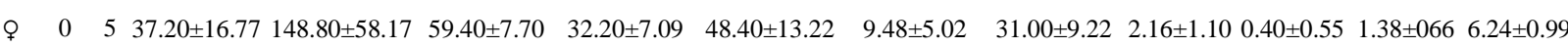

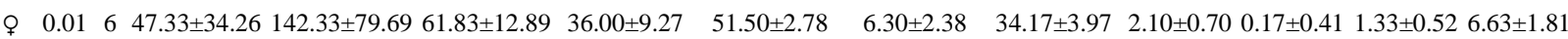

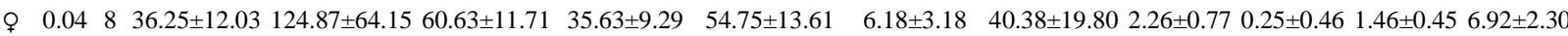

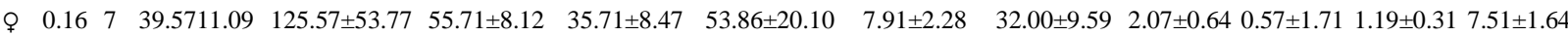

ㅇ $\quad 0.64 \quad 6 \quad 26.3315 .12 \quad 108.67 \pm 52.67 \quad 56.33 \pm 10.10 \quad 33.83 \pm 8.91 \quad 74.67 \pm 15.76 * \quad 5.02 \pm 1.59 \quad 40.50 \pm 1.56 \quad 1.90 \pm 0.67 \quad 0.17 \pm 0.41 \quad 1.33 \pm 0.38 \quad 6.75 \pm 2.49$

Alanine aminotransferase (ALT), aspartate aminotransferase (AST), total protein (TP), albumin (ALB), serum uric acid (UA), blood urea nitrogen

(BUN), creatinine (CR), total cholesterol (TC), triglyceride (TG), high-density lipoprotein-cholesterol (HDL-C) and glucose(GLU)

*. Statistical significance: $\mathrm{p}<0.05$, compared with normal control.
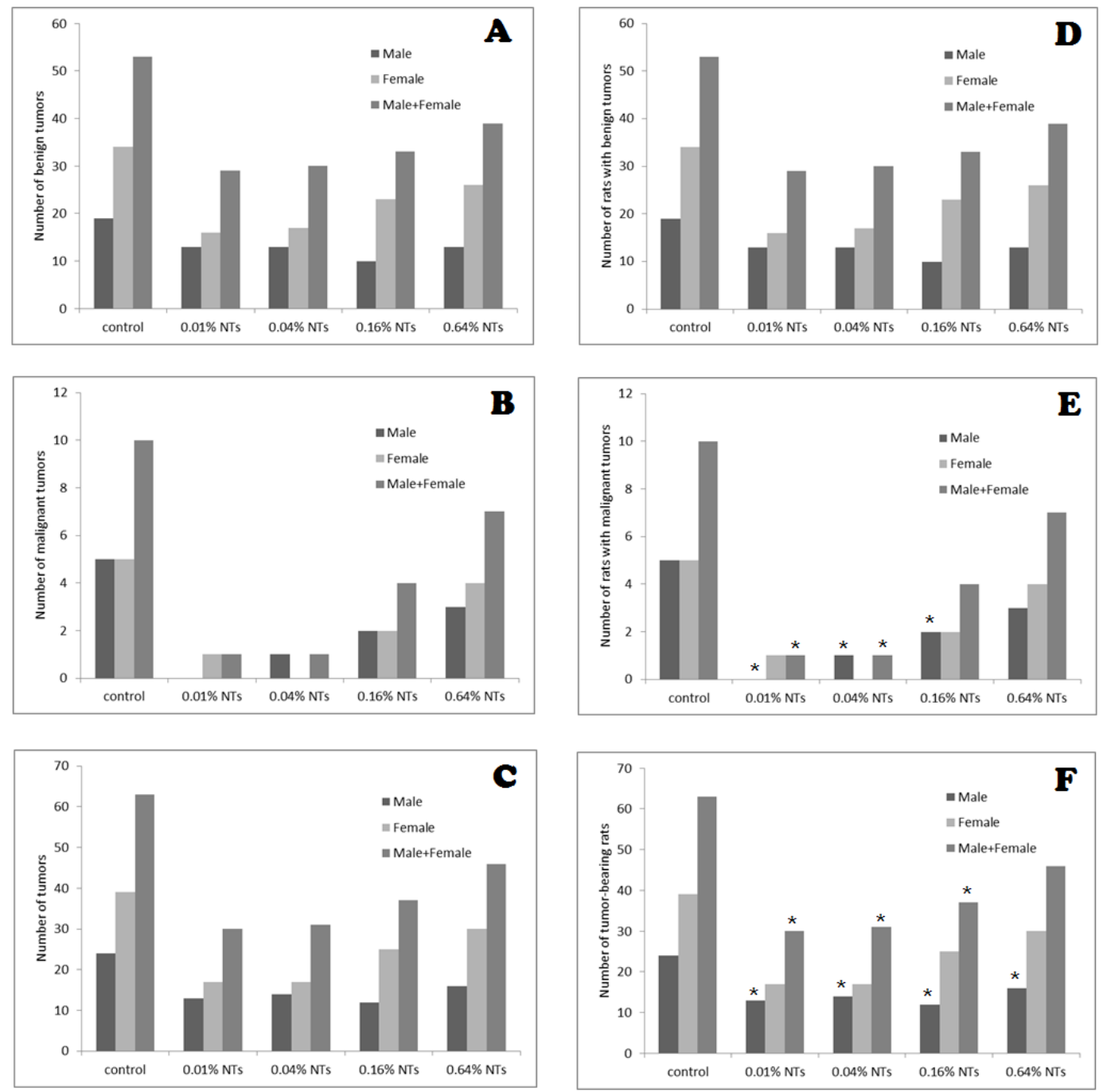

Figure 1. Effect of NTs on spontaneous neoplastic lesions trendsin Sprague-Dawley rats (male, female and gender pooled) treated with different levels of dietary nucleotides, shown by the number of benign tumors (A), the number of malignant tumors (B), tumors (C), the number of rats with benign tumors (D), the number of rats with malignant tumors (E) and the number of tumor-bearing rats (F)

Significant difference compared with the control group (by Fisher's exact probability test): *P $<0.05$. 
Table 3. Primary causes of spontaneous deaths of male Srague-Dawley rats treated with different levels of dietary nucleotides Male

Death cause NTs (\%)

\begin{tabular}{|c|c|c|c|c|c|c|c|}
\hline \multirow{2}{*}{$\begin{array}{c}\text { Male } \\
\text { Death cause }\end{array}$} & \multicolumn{5}{|c|}{ NTs (\%) } & \multirow{2}{*}{$\chi^{2}$} & \multirow{2}{*}{$\mathrm{P}$} \\
\hline & $0(n=26)$ & $0.01(\mathrm{n}=26)$ & $0.04(\mathrm{n}=26)$ & $0.16(\mathrm{n}=26)$ & $0.64(\mathrm{n}=26)$ & & \\
\hline \multirow{2}{*}{\multicolumn{8}{|c|}{ Neoplastic lesion }} \\
\hline & & & & & & & \\
\hline Pituitary gland & 6 & 3 & 2 & 3 & 3 & 2.78 & 0.648 \\
\hline Skin/subcuits & 7 & 5 & 3 & 4 & 6 & 2.476 & 0.649 \\
\hline Subtotal & 13 & 8 & 5 & 7 & 9 & 6.19 & 0.185 \\
\hline \multicolumn{8}{|l|}{ Malignant } \\
\hline Lung & 1 & & & & & & \\
\hline Pancreas & & & & 1 & 2 & & \\
\hline Adrenal gland & & & & 1 & & & \\
\hline Bladder & 1 & & 1 & & & & \\
\hline Abdominal metastases & 1 & & & & 1 & & \\
\hline Leukemia & 2 & & & & & & \\
\hline Subtotal & 5 & 0 & 1 & 2 & 3 & 6.67 & 0.134 \\
\hline Total & 18 & $8^{*}$ & $5 *$ & 9* & 12 & 15.58 & 0.004 \\
\hline \multicolumn{8}{|l|}{ Non-neoplastic lesion } \\
\hline Chronic nephropathy & 2 & 3 & 3 & 3 & 1 & 1.68 & 0.891 \\
\hline Emphysema & & 1 & 1 & 2 & 2 & 2.59 & 0.875 \\
\hline Hepatocirrhosis & 1 & 2 & 2 & 2 & 3 & 1.29 & 0.986 \\
\hline Hepatic steatosis & 2 & 3 & 4 & 3 & 3 & 0.93 & 0.993 \\
\hline Megalosplenia & & 1 & 1 & 1 & & & \\
\hline Intestinal obstruction & & 1 & 2 & & & & \\
\hline Diabetes & & 2 & 2 & 1 & 1 & 2.57 & 0.875 \\
\hline Total & 5 & 13 & 15 & 12 & 10 & 9.14 & 0.058 \\
\hline Undetermined & 3 & 5 & 5 & 5 & 4 & 0.875 & 0.928 \\
\hline
\end{tabular}

Data are the numbers of deaths.

Significant difference compared with the control group (by Fisher's exact probability test):* $\mathrm{P}<0.0125$

Table 4. Primary causes of spontaneous deaths of female Srague-Dawley rats treated with different levels of dietary nucleotides

\begin{tabular}{|c|c|c|c|c|c|c|c|}
\hline \multirow{2}{*}{$\begin{array}{c}\text { Female } \\
\text { Death cause }\end{array}$} & \multicolumn{5}{|c|}{ NTs (\%) } & \multirow{2}{*}{$x^{2}$} & \multirow{2}{*}{$\mathrm{P}$} \\
\hline & $0(n=26)$ & $0.01(n=26)$ & $0.04(n=26)$ & $0.16(n=26)$ & $0.64(n=26)$ & & \\
\hline \multicolumn{8}{|l|}{ Neoplastic lesion } \\
\hline Pituitary gland & 5 & 4 & 5 & 7 & 6 & 1.22 & 0.876 \\
\hline Mammary gland & 4 & 4 & 2 & 5 & 5 & 1.94 & 0.787 \\
\hline Skin/subcuits & 2 & 1 & 1 & & 1 & 2.24 & 0.958 \\
\hline Subtotal & 11 & 9 & 8 & 12 & 12 & 2.12 & 0.715 \\
\hline \multicolumn{8}{|l|}{ Malignant } \\
\hline Mammary gland & 2 & & & & 1 & & \\
\hline Lung & 1 & & & & 1 & & \\
\hline Pancreas & 1 & & & 1 & 1 & & \\
\hline Ovarian & & & & & 1 & & \\
\hline Abdominal metastases & 1 & 1 & & 1 & & & \\
\hline Subtotal & 5 & 1 & 0 & 2 & 4 & 7.75 & 0.066 \\
\hline Total & 16 & 10 & 8 & 14 & 16 & 8.13 & 0.087 \\
\hline \multicolumn{8}{|l|}{ Non-neoplastic lesion } \\
\hline Chronic nephropathy & 1 & 1 & 2 & 1 & 1 & 1.10 & 1.000 \\
\hline Emphysema & & 2 & 2 & 1 & & 3.71 & 0.559 \\
\hline Hepatocirrhosis & 1 & 2 & 3 & 1 & 1 & 2.02 & 0.857 \\
\hline Hepatic steatosis & 2 & 3 & 4 & 3 & 3 & 0.93 & 0.993 \\
\hline Megalosplenia & 2 & 1 & & 1 & 1 & 2.24 & 0.958 \\
\hline Intestinal obstruction & & 1 & 1 & & & 3.02 & 1.000 \\
\hline Diabetes & 1 & 1 & 2 & 1 & & 2.24 & 0.958 \\
\hline Total & 7 & 11 & 14 & 8 & 6 & 7.20 & 0.126 \\
\hline Undetermined & 3 & 5 & 5 & 4 & 4 & 0.93 & 0.981 \\
\hline
\end{tabular}

Data are the numbers of deaths. 